\title{
Transverse optical plasmons in layered superconductors.
}

\author{
D. van der $\mathrm{Marel}^{a}$,and A. Tsvetkov ${ }^{a, b}$ \\ ${ }^{a}$ Materials Science Center, Laboratory of Solid State Physics, University of Groningen, \\ Nijenborgh 4, 9747 AG Groningen, The Netherlands \\ ${ }^{b}$ P. N. Lebedev Physical Institute, Russian Academy of Sciences, Leninsky prospect 53, 117924 Moscow, \\ Russia
}

We discuss the possible existance of transverse optical plasma modes in superlattices consisting of Josephson coupled superconducting layers. These modes appear as resonances in the current-current correlation function, as opposed to the usual plasmons which are poles in the density-density channel. We consider both bilayer superlattices, and single layer lattices with a spread of interlayer Josephson couplings. We show that our model is in quantitative agreement with the recent experimental observation by a number of groups of a peak at the Josephson plasma frequency in the optical conductivity of $\mathrm{La}_{1.85} \mathrm{Sr}_{0.15} \mathrm{CuO}_{4}$.

\section{INTRODUCTION}

In recent years interlayer Josephson plasmons have received considerable attention both theoretically and experimentally. So far this attention has been restricted to systems consisting of a stack of identical 2-dimensional superconducting layers coupled by identical insulating barriers. In this paper we consider the more general problem of ordered and disordered superlattice structures. Regardless of the underlying microscopic model, in the superconducting state the low energy scale properties of a multilayer of Josephson coupled 2-dimensional superconducting layers is described by the Lawrence-Doniach model[1]. This model has been applied to the case of an ordered array of single superconducting layers, by Bulaevskii et al.[2], who calculated the longitudinal dielectric function, and by Tachiki et al. [3], who studied the transverse dielectric function. Microscopically the interlayer Josephson resonances are a result of broken gauge symmetry in a superconductor with long range Coulomb interactions[4], and belong to a larger class of several types of collective modes in layered unconventional superconductors[5]. A low frequency Josephson plasmon[6] arises as a direct consequence of the electrodynamical properties of a BCS superconductor in the dirty limit $[7,8,9]$. A different scenario has been discussed by Anderson, in which these modes occur as a direct consequence of the interlayer pairing mechanism for superconductivity in the high $\mathrm{T}_{c}$ materials $[10,11]$. In this spirit we attempt to set the stage for further experimental studies of layered superconductors, in particular materials with more than one superconducting layer per unit cell (e.g. $\mathrm{Bi}_{2} \mathrm{Sr}_{2} \mathrm{CaCu}_{2} \mathrm{O}_{8}$ ) and materials where the interlayer Josephson parameters are randomly distributed due to disorder (e.g. $\left.\mathrm{La}_{2-x} \mathrm{Sr}_{x} \mathrm{CuO}_{4}[6,7]\right)$.

\section{RESULTS}

To simplify the discussion we will only consider the charge dynamics for $\vec{k} \rightarrow 0$, with the electric field vector perpendicular to the $\mathrm{CuO}_{2}$ planes, which is the relevant limit for a discussion of the $c$-axis optical properties. A discussion of the dielectric tensor for general values of $k$ was given by Bulaevskii for the superconducting state[2] and by one of us for the normal state[12]. Let us consider the relevant Hamiltonian of a stack of superconducting planes coupled through the Josephson effect, each with a total area $A$, and with total thickness $D$ of the crystal along the $z$ direction.

Each plane is characterized by a charge per unit area $\rho_{m}$ and a phase $\phi_{m}$, where $m$ is the layer index. As we are only considering electrical fields perpendicular to the planes, the long-range Coulomb potential between each set of planes increases linearly with distance. The charge dynamics enters via the Josephson coupling $J_{m}$ between each set of nearest neighbor planes. The equations of motion for a general distribution of interlayer couplings, and general values of wavevector $\vec{k}$ were derived by Bulaevskii[2]. In the present paper we only consider $\vec{k} \rightarrow 0$, relevant for 
optical experiments. The solution for general values of $\vec{k}$ will be presented elsewhere[13]. The limit $\vec{k} \rightarrow 0$ leads to a considerable simplification of the equations of motion, which in this case correspond exactly to an equivalent network model where each set of two neighbouring layers can be represented by a capacitance shunted by an induction. The total complex impedance $Z$ of the crystal is the series resistance of all local impedances $Z_{m}=4 \pi i d_{m} /\left(A \omega \epsilon_{m}\right)$, where we introduce the quantity $\epsilon_{m} \equiv 1-\omega_{J, m}^{2} / \omega^{2}$. Here the screened Josephson plasma frequency is defined as $\omega_{J, m}^{2}=4 \pi \Phi_{0}^{-1} \epsilon_{\infty}^{-1} d_{m} J_{m}$, and $d_{m}$ is the distance between neighboring planes. The expression for the macroscopic dielectric function $\epsilon$ follows immediately after identifying $Z A / D$ with the complex conductivity $1 / \sigma$, and by using $\epsilon=4 \pi i \sigma / \omega$. This model can be easily extended to take into account quasi-particle currents by shunting each $Z_{m}$ with a resistor in series with an induction, resulting in an expression for $\epsilon$ analogous to the GorterCasimir two-fluid model, with a normal-fluid component $\sigma_{n}=\sigma_{0} /(1-i \omega \tau)$. Polarization of the ions and transverse optical phonons can be incorporated by adding separate oscillators to the expression for $\epsilon$. The expression for $\epsilon$ becomes

$$
\epsilon=\left(\sum_{m} \frac{w_{m}}{\epsilon_{m}}\right)^{-1}
$$

where $w_{m}=d_{m} / D$ is a weighting factor proportional to the distance between the layers. The total dielectric function is a sum over response functions of sets of two coupled planes for which we obtain

$$
\epsilon_{m}=\epsilon_{\infty}\left(1-\frac{\omega_{J, m}^{2}}{\omega^{2}}\right)+\frac{4 \pi i}{\omega} \sigma_{n}
$$

\section{BILAYER SUPERCONDUCTORS}

Let us now consider a crystal which has two superconducting layers per unit cell. We denote the Josephson coupling constants as I and $\mathrm{K}$, the distances between the planes as $d_{I}$ and $d_{K}$, and the screened Josephson plasma frequencies as $\omega_{I}$ and $\omega_{K}$. Using Eq. (3), and approximating the effect of ionic and vibrational screening with a constant $\epsilon_{\infty}$, the dielectric function is

$$
\epsilon=\frac{\epsilon_{\infty}\left(\omega^{2}-\omega_{I}^{2}\right)\left(\omega^{2}-\omega_{K}^{2}\right)}{\omega^{2}\left(\omega^{2}-\omega_{T}^{2}\right)}
$$

where we introduced $\omega_{T}^{2}=\left(d_{I} \omega_{K}^{2}+d_{K} \omega_{I}^{2}\right) /\left(d_{I}+\right.$ $\left.d_{K}\right)$. Interestingly, there are now two longitudinal plasmons. Also the dielectric function has a pole at $\omega_{T}$, which corresponds to a transverse optical plasma oscillation, with oscillator-strength $S_{T}=$ $\epsilon_{\infty}\left(1-\omega_{I}^{2} / \omega_{T}^{2}\right)\left(1-\omega_{K}^{2} / \omega_{T}^{2}\right)$. Photons with frequen-

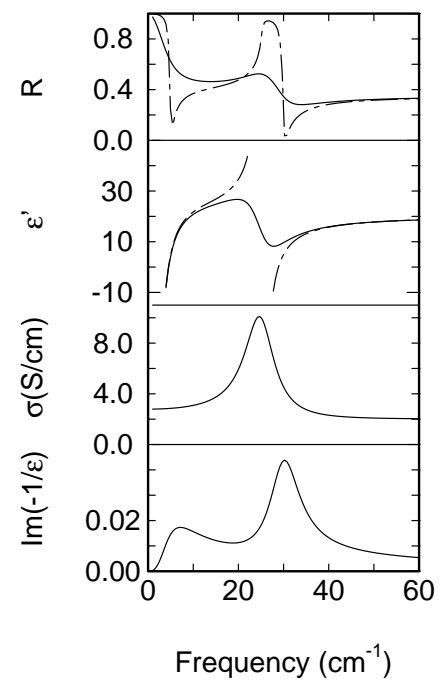

Figure 1: Reflectivity, Re $\epsilon$, optical conductivity and loss function calculated for a hypothetical bi-layer superconductor, assuming $\omega_{I}=5 \mathrm{~cm}^{-1}, \omega_{T}=26 \mathrm{~cm}^{-1}$, and $\omega_{K}=30 \mathrm{~cm}^{-1}$. The chained curves were calculated assuming no normal-fluid component in the conduction, the solid curves were calculated assuming a parallel conductance of $2 \mathrm{~S} / \mathrm{cm}$.

cies within the Reststrahlenband $\omega_{T}<\omega<\omega_{K}$, and with $\vec{E}$ polarized along $z$ can not propagate inside the crystal and are totally reflected at the interface. Such a reflectivity spectrum of the crystal-face perpendicular to the superconducting layers is displayed in Fig. 1 , along with the dispersion relation. We see, that a lifting of degeneracy between the two Josephson frequencies $\omega_{I}$ and $\omega_{K}$ should be easily recognizable in the reflectivity curve, even if the features in $\sigma$ are blurred due to parallel conduction of quasi-particles. The compound $\mathrm{Bi}_{2} \mathrm{Sr}_{2} \mathrm{CaCu}_{2} \mathrm{O}_{8}$ may exhibit such behaviour. There a resonance found at $5 \mathrm{~cm}^{-1}[14,15]$ is probably the lowest of the two Josephson plasmons. The higher plasma frequency has not yet been observed with infrared spectroscopy down to $\approx 30$ $\mathrm{cm}^{-1}[16]$ and can be situated anywhere between 5 and $30 \mathrm{~cm}^{-1}$. 


\section{SINGLE LAYER SUPERCONDUCTORS}

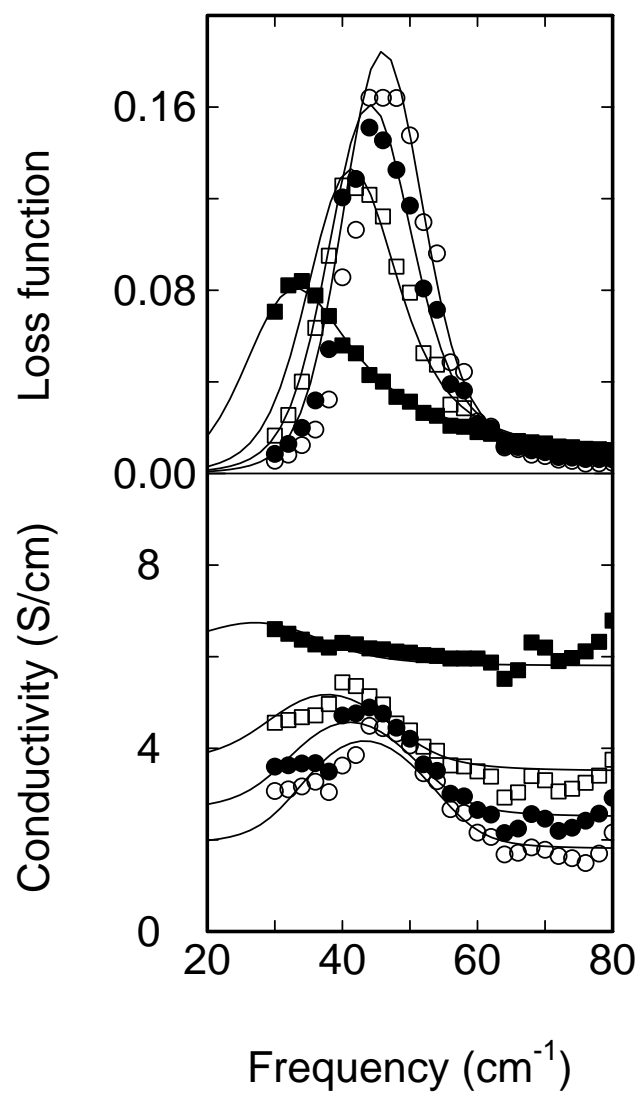

Figure 2: Experimental loss function (upper panel) and conductivity (lower panel) of $\mathrm{La}_{1.85} \mathrm{Sr}_{0.15} \mathrm{CuO}_{4}$ at $10 \mathrm{~K}$ (open circles), $15 \mathrm{~K}$ (close circles), $20 \mathrm{~K}$ (open squares), and $27 \mathrm{~K}$ (closed squares). The solid lines are a fit with the same relative gaussian distribution with a FWHM which is $20 \%$ of the centerfrequency.

We now apply Eq. 1 to a stack of one superconducting layer per unit cell, but with a distribution of the interlayer coupling around a center value. As was the case for the ordered superlattices discussed above, the material is effectively homogeneous vis $a$ vis the optical response of a lightbeam reflecting on the $a c$-face of the crystal, as long as the length scale of variations of $\epsilon_{m}$ is much smaller than the wavelength of the electromagnetic radiation. Let us now consider the case where a spread exists of the Josephson frequencies around a center frequency. The source of such could be the local variations in the potential barrier, e.g. due to random substition of $\mathrm{La}^{3+}$ with $\mathrm{Sr}^{2+}$ ions in the tunneling barrier between the superconducting $\mathrm{CuO}_{2}$ layers in $\mathrm{La}_{2-x} \mathrm{Sr}_{x} \mathrm{CuO}_{4}$. The expression for $\epsilon$ then becomes

$$
\frac{1}{\epsilon}=\int d \omega_{J} \frac{\rho\left(\omega_{J}\right) \omega^{2}}{\epsilon_{\infty}\left(\omega^{2}-\omega_{J}^{2}\right)+4 \pi i \omega \sigma_{n}}
$$

where $\rho\left(\omega_{J}\right)$ is the normalized distribution function of the screened Josephson plasma frequencies. The distribution $\rho\left(\omega_{J}\right)$ should decay sufficiently rapidly for $\omega_{J} \rightarrow 0$. Physically this corresponds to the condition that the weakest Josephson junctions in the stack of layers should still have a finite critical current (or $\omega_{J}$ value).

A peak in the optical conductivity which coincides with the longitudinal Josephson plasma has been reported by at least four different groups $[6,17,7,18]$, but has to our knowledge not been explained in a satisfactory way. In Fig. 2 we display both the experimental loss function and the conductivity for the region near the Josephson plasma frequency. These data were adopted from the paper by Kim et al.. So far we had the data of Gerrits et al. and Kim et al. at our disposal, and checked the validity of our approach for all temperatures reported for these two sets of data. The agreement with the formalism outlined above is very satisfactory at all temperatures. To demonstrate this result we display in the top panel of Fig. 2 the loss function at $10 \mathrm{~K}$ of Kim et al.. In the lower panel the optical conductivity is displayed. Clearly both the loss function and $\sigma$ peak at the same frequency of $48 \mathrm{~cm}^{-1}$. If we assume that the 'intrinsic' value of the $c$-axis conductivity is 1.8 $\mathrm{S} / \mathrm{cm}$ in this case (dashed line in the lower panel), and assuming that there is a single value of $\omega_{J}$ of $48 \mathrm{~cm}^{-1}$, we obtain the dashed curve for the loss function in the top panel. The finite width in this case is due to the presence of a finite background conductivity, and is given by $2 / \tau=8 \pi \sigma_{n} / \epsilon_{\infty}$. With $\sigma_{n}=1.8 \mathrm{~S} / \mathrm{cm}$ and $\epsilon_{\infty} \approx 22$ we obtain $c / \tau=2 \pi \cdot 10$ $\mathrm{cm}^{-1}$. In other words: Experimentally the broadening of the plasmon-peak can not be explained as the usual lifetime effect. If we now assume that in addition $\omega_{J}$ has a Gaussian distribution, we obtain the solid and chained curves in Fig. 2 for the loss function and the conductivity using Eq. 4 with a FWHM of 11 and $17 \mathrm{~cm}^{-1}$ respectively. Clearly the choice of FWHM which gives good agreement for the loss function, also explains the anomalous peak in the conductivity both qualitatively and quantitatively. 


\section{CONCLUSIONS}

We discussed the possible existance of transverse optical Josephson plasmons in layered superconductors with 2 or more layers per unit cell. A related -but not similar- effect effect may have already been observed in $\mathrm{La}_{2-x} \mathrm{Sr}_{x} \mathrm{CuO}_{4}$ due to variations in the interlayer Josephson frequency, possibly due to the random substitution of La with Sr. It should be possible to design and grow artificial superconducting multilayers with optical properties taylored to specific needs in the $\mathrm{THz}$ range. Because they provide an effective channel for coupling electromagnetic radiation to the current perpendicular to the superconducting layers, transverse optical Josephson plasmons may become useful for the detection and emission of far-infrared radiation in the $\mathrm{THz}$ range.

\section{REFERENCES}

[1] W. E. Lawrence and S. Doniach in Proceedings of the 12th International Conference on Low Temperature Physics, Kyoto, 1970, edited by E. Kanda (Keigaku, Tokyo, 1970); S. Doniach and M. Inui, Phys. Rev. B 41, 6668 (1990).

[2] L. N. Bulaevskii et al., Phys. Rev. B. 50, 12831 (1994).

[3] M. Tachiki, T. Koyama and S. Takahaski, Phys. Rev. B 50, 7065 (1994).

[4] P. W. Anderson, Phys. Rev. 112, 1900 (1958).

[5] D. van der Marel, Phys. Rev. B 51, 1147 (1995).

[6] K. Tamasaku, Y. Nakamura and S. Uchida, Phys. Rev. Lett. 69, 1455 (1992).

[7] J. H. Kim et al., Physica C 247, 297 (1995).

[8] S. V. Pokrovsky and V. L. Pokrovsky, J. of Superconductivity 8, 183 (1995).

[9] Y. Zha, K. Levine, and D. Z. Liu, Phys. Rev. B 51, 6602 (1995).

[10] S. Chakravarty and P. W. Anderson, Phys. Rev. Lett. 72, 3859 (1994).

[11] P.W. Anderson, Science 268, 1154 (1995).

[12] D. van der Marel and J. H. Kim, J. Phys. Chem. Solids 561825 (1995).

[13] D. van der Marel, unpublished.

[14] Y.Matsuda et al., Phys. Rev. Lett. 75, 4512 (1995).

[15] O. K. C. Tsui, N. P. Ong and J. B. Peterson, Phys. Rev. Lett. 76, 819 (1996).

[16] S. Tajima et al., Phys. Rev. B 48, 16164 (1993).

[17] A. M. Gerrits et al., Phys. Rev. B 51, 12049 (1995).

[18] D. N. Basov, et al., Phys. Rev. B 52, 13141 (1995). 